\title{
Levetiracetam Induced Hypoglycemia - A Case Report
}

\author{
Authors \\ Azma Naaz Mohammed ${ }^{1 *}$, Narayana Geetha T.L ${ }^{2}$, Sundar Raju Y.G ${ }^{3}$ \\ *Corresponding Author
}

Azma Naaz Mohammed

\begin{abstract}
Hypoglycemia is a clinical syndrome with diverse causes in which low blood sugars lead to signs and symptoms thereby exposing an individual to harm. While it is a known entity in diabetics on glucose lowering drugs, hypoglycaemia in non diabetics can be caused by a variety of non diabetic drugs, including alcohol. Here we report such case of a 20 year old male patient, who was admitted with seizures and developed hypoglycaemia with levetiracetam, which was dose dependent and persistent, only to be subsiding after substitution with oxcarbazepine. Adverse effects of any drug which we know from studies and books only account to around 35\% and still newer side effects can be resurfaced and encountered in our day to day practice of medicine. This article is an effort to report association of dose dependent hypoglycaemia with levetiracetam and also a step towards encouraging the adverse effect-reporting.
\end{abstract}

\section{Introduction}

Hypoglycemia is a clinical syndrome with diverse causes in which low blood sugars lead to signs and symptoms thereby exposing an individual to harm. While it is a known entity in diabetics on glucose lowering drugs, hypoglycaemia in non diabetics can be caused by a variety of non diabetic drugs, including alcohol and common critical illness such as hepatic, renal or cardiac failure, sepsis or inanition. While wide number of drugs keep on entering our medical armamentarium every day, rarer and newer side effects spring out. Therefore, the primary task in a patient without diabetes is to make an accurate diagnosis of what may be the cause of hypoglycaemia. Due to lack of databases and adverse effect-reporting, we still see adverse effects which are often less predictable and unexpected. Here we report such case of a 20year old male patient, who was admitted with seizures and developed hypoglycaemia with levetiracetam, which was dose dependent and persistent, only to be subsiding after substitution with oxcarbazepine.

\section{Case Report}

A 20 year old male patient was admitted with complaints of 6-7 episodes of stiffening of body, uprolling of eye balls associated with loss of consciousness from morning on the day of admission. He also gives one similar episode one month back, which he neglected. He was evaluated and treated on lines of status epilepticus. His blood investigations revealed $\mathrm{Hb}-10.1 \mathrm{gm} / \mathrm{dl}$, TLC 9800 cells $/ \mathrm{mm}^{3}$, Calcium - 8.78 (Ionised $\mathrm{Ca}^{2+}$ 5.54), S.Creatinine - $0.697 \mathrm{mg} / \mathrm{dl}$, Bl. Urea - 12.41 $\mathrm{mg} / \mathrm{dl}$, Sodium - $140 \mathrm{mEq} / \mathrm{L}$, Potassium - 3.85 $\mathrm{mEq} / \mathrm{L}, \mathrm{Mg}^{2+}-2.0 \mathrm{mg} / \mathrm{dl}, \mathrm{RBS}-104 \mathrm{mg} / \mathrm{dl}$, SGOT 19, SGPT -14. His MRI of Brain revealed no abnormalities. EEG showed generalised abnormally drowsy reading with background slowing 
suggestive of GTCS. He was loaded with Phenytoin and was given $100 \mathrm{mg}$ iv thrice daily. However when he had another seizure on the following day, he was loaded with Levetiracetam $1 \mathrm{gm}$ followed with maintenance dose of $500 \mathrm{mg}$ iv thrice daily added to phenytoin. After 6hrs after loading dose of Levetiracetam, he developed sweating and anxiety when his RBS was found to be $47 \mathrm{mg} / \mathrm{dl}$. His blood sugars were charted and was kept on $25 \%$ Dextrose infusion, despite of which his RBS was in the range of $58-87 \mathrm{mg} / \mathrm{dl}$. At another episode of hypoglycaemia, his Insulin levels were measured CT abdomen done to rule out any Insulinoma. The CT scan was normal and Insulin level was 14.6 $\mathrm{mIU} / \mathrm{L}$ (normal range 2-25mIU/L). His HbA1c was 5.0 ruling out any previous hypoglycaemia. When his Levetiracetam dose was reduced to twice daily and was shifted to oral route, his blood sugars stabilised around $90-110 \mathrm{mg} / \mathrm{dl}$ on DNS continuous infusion. Then Levetiracetam was gradually tapered and was substituted by oxcarbazepine. His blood sugars became normal and when he had no recurrence of seizure, he was discharged.

\section{Discussion}

Drug induced hypoglycaemia is a significant adverse effect that may cause morbidity and must be included in the differential diagnosis of hypoglycaemia. In one study, drug-induced hypoglycemia represented $23 \%$ of all hospital admissions attributed to adverse drug events, which in turn represented $4.4 \%$ of all admissions ${ }^{1}$. Although most cases of drug-induced hypoglycemia were described in patients with diabetes taking glucose-lowering treatments, particularly sulfonylureas and insulin, several other nondiabetic pharmacological agents have also been implicated ${ }^{2}$. A survey of safety reporting in 192 randomized drug trials demonstrated that the severity of clinical and laboratory adverse effects was adequately defined in only 39 and $29 \%$ of trial reports, respectively ${ }^{3}$. Seltzer ${ }^{2}$ found that only 1418 drug induced hypoglycaemia cases were reported in the literature between 1940 and 1989, which is likely an underestimate.
Levetiracetam, with an oxypyrrollidine ring in its structure, is an antiepileptic drug prescribed for focal onset seizures and as adjuvant therapy in tonic-clonic seizures, partial and myoclonic seizures. Owing to negligible side effects and no interaction with other drugs, it is widely popular among neurologists. The reported side effects, though less common are sedation, fatigue, in coordination and mood changes. Hypoglycemia is not a side effect mentioned in the drug information or in any trials. In their article, Kristen Helms and Kristi Kelley, mentioned a list of non glucose lowering drugs responsible for hypoglycaemia, where Levetiracetam doesn't find a place. However an article from French pharmacovigilance database ${ }^{5}$ reveals hypoglycaemia with Levetiracetam exposure in 5 patients -2 newborns(both female) who had hypoglycaemia born to mothers who were on Levetiracetam in pregnancy; 2 male patients who along with levetiracetam also took valproate through unspecified route had hypoglycaemia only to be resolved after discontinuation of levetiracetam and another 1 male patient who had hypoglycaemia with parenteral route which did not recur with oral route. The case reports mentioned were difficult to analyse due to valproate association in 3 out of 5 patients and retrospective nature of the report makes us difficult to elucidate any other details for the association between hypoglycaemia and levetiracetam.

In our patient, Hypoglycemia occurred 6 hours after administration of levetiracetam, persisted despite glucose supplementation, decreased when dose was reduced and route of administration was changed. However hypoglycaemia got resolved only after 1 day of stopping of the drug. This throws light on the fact that hypoglycaemia due to levetiracetam was Dose dependent and was more with parenteral route than oral route in our patient. But the mechanism by which this occurred is unclear as literature is not available regarding any possible mechanism by which oxypyrollidine of levetiracetam could be responsible for hypoglycaemia. 


\section{Conclusion}

In conclusion, adverse effects of any drug which we know from studies and books only account to around $35 \%$ and still newer side effects can be resurfaced and encountered in our day to day practice of medicine. This article is an effort to report association of dose dependent hypoglycaemia with levetiracetam and also a step towards encouraging the adverse effect-reporting.

\section{References}

1. Kilbridge PM, Campbell UC, Cozart HB, Mojarrad MG 2006 Automated surveillance for adverse drug events at a community hospital and an academic medical center. $J$ Am Med Inform Assoc 13:372-377

2. Seltzer

HS 1989 Drug-induced hypoglycaemia A review of 1418 cases. Endocrinol Metab Clin North Am 18: 163-183

3. Ioannidis JP, Lau J 2001 Completeness of safety reporting in randomized trials: an evaluation of 7 medical areas. JAMA 285:437-443

4. Kristen Helms and Kristi Kelley (2011). Drug-Induced Hypoglycemia, Hypoglycemia - Causes and Occurrences, Prof.EverlonRigobelo (Ed.), InTech. 